\title{
NEWTON SUM RULES AND MONOTONICITY PROPERTIES OF THE ZEROS OF SCALED CO-RECURSIVE ASSOCIATED POLYNOMIALS
}

\author{
E. K. Ifantis, C. G. Kokologiannaki, and P. D. Siafarikas
}

Dedicated to Prof. Lee Lorch for his 80th birthday

$$
\begin{aligned}
& \text { ABSTRACT. Let } Q_{n}(x ; \beta, \gamma, c) \text { be the polynomials of degree } n \text { which satisfy the } \\
& \text { recurrence relation: } \\
& \begin{array}{r}
\alpha_{n+c} Q_{n+1}(x ; \beta, \gamma, c)+ \\
\qquad \alpha_{n+c-1} Q_{n-1}(x ; \beta, \gamma, c)+\left(\beta_{n+c}+\beta \delta_{n, 0}\right) Q_{n}(x ; \beta, \gamma, c) \\
=x\left(1+(\gamma-1) \delta_{n, 0}\right) Q_{n}(x ; \beta, \gamma, c), \\
Q_{-1}(x ; \beta, \gamma, c)=0, \quad Q_{0}(x ; \beta, \gamma, c)=1 .
\end{array}
\end{aligned}
$$

In the above, $\beta$ is real, $\gamma>0, \alpha_{n+c}$ and $\beta_{n+c}$ are real sequences with $\alpha_{n+c}>0$, and $\delta_{n, 0}$ is the Kronecker symbol. These polynomials are called scaled co-recursive associated polynomials. The co-recursive associated orthogonal polynomials are obtained from the above for $\gamma=1$.

In this paper, the Newton sum rules for the $k$-th power of the zeros of scaled co-recursive associated orthogonal polynomials are determined in terms of the Newton sum rules of associated orthogonal polynomials. Some monotonicity properties of the zeros also are given.

\section{Introduction}

The orthogonal polynomials $P_{n}(x), n \geq 0$, of degree $n$, with respect to a positive Borel measure on the real line with infinite mass points, can be defined by the recurrence relation:

$$
\begin{gathered}
\alpha_{n} P_{n+1}(x)+\alpha_{n-1} P_{n-1}(x)+\beta_{n} P_{n}(x)=x P_{n}(x) \\
P_{-1}(x)=0, \quad P_{0}(x)=1,
\end{gathered}
$$

where $\alpha_{n}>0$ and $\beta_{n}$ are real sequences.

The associated polynomials $P_{n}(x ; c)$ of the above polynomials are obtained when we replace $n$ by $n+c$ in the coefficients $\alpha_{n}$ and $\beta_{n}$ of (1.1), i.e.,

$$
\begin{gathered}
\alpha_{n+c} P_{n+1}(x ; c)+\alpha_{n+c-1} P_{n-1}(x ; c)+\beta_{n+c} P_{n}(x ; c)=x P_{n}(x ; c), \\
P_{-1}(x ; c)=0, \quad P_{0}(x ; c)=1 .
\end{gathered}
$$

The associated polynomials are called, associated of order $c$ if $c$ is an integer and numerator polynomials if $c=1$. For particular cases of associated polynomials, many authors $[3,4,17,18,37,38,43]$ gave explicitly the corresponding 4th-order differential equations. Very recently [46] for the associated polynomials of arbitrary order $c$ which satisfy (1.2), the corresponding unique differential equation was calculated with the help of a program built in Mathematica symbolic language. Results for particular

Received September 19, 1995, revised July 9, 1996.

1991 Mathematics Subject Classification: 33C10.

Key words and phrases: scaled co-recursive associated polynomials, zeros, Newton sum rules, monotonicity. 
cases of associated polynomials concerning explicit forms, orthogonality measures, monotonicity properties, and differential inequalities for their zeros were given in [2, $18,19,24,28,32,43]$.

The co-recursive polynomials $Q_{n}(x)$ of $P_{n}(x)$, which first were introduced by T. S. Chihara [8], satisfy the same recurrence relation (1.1) with the restriction $Q_{1}=$ $\frac{x-\beta_{0}-\beta}{\alpha_{0}}$. They can be defined directly by

$$
\begin{gathered}
\alpha_{n} Q_{n+1}(x)+\alpha_{n-1} Q_{n-1}(x)+\left(\beta_{n}+\beta \delta_{n, 0}\right) Q_{n}(x)=x Q_{n}(x) \\
Q_{-1}(x)=0, \quad Q_{0}(x)=1
\end{gathered}
$$

where

$$
\delta_{n, 0}= \begin{cases}1 & \text { for } n=0 \\ 0 & \text { for } n \neq 0\end{cases}
$$

Orthogonal polynomials with perturbed recurrence relations play an important role not only with the Orthogonal Polynomials Theory (see $[1,8,33,34,39]$ ), but also in some other areas such as Quantum Mechanics [42], Birth and Death Processes [27], etc. Recently A. Ronveaux and F. Marcellan [39] gave explicitly the 4th-order differential equation satisfied by the co-recursive polynomials of the classical orthogonal polynomials.

The scaled co-recursive associated polynomials satisfy the recurrence relation

$$
\begin{gathered}
\alpha_{n+c} Q_{n+1}(x ; \beta, \gamma, c)+\alpha_{n+c-1} Q_{n-1}(x ; \beta, \gamma, c) \\
+\left(\beta_{n+c}+\beta \delta_{n, 0}\right) Q_{n}(x ; \beta, \gamma, c)=x\left(1+(\gamma-1) \delta_{n, 0}\right) Q_{n}(x ; \beta, \gamma, c), \\
Q_{-1}(x ; \beta, \gamma, c)=0, \quad Q_{0}(x ; \beta, \gamma, c)=1,
\end{gathered}
$$

where $\gamma>0$. For $\gamma=1$ these polynomials are the co-recursive associated orthogonal polynomials, which were introduced by J. Letessier in $[29,30,31]$ for special cases. The case $\gamma=0$ is also of some interest in connection with the results in $[13,14,21$, 41].

Given an orthogonal polynomial family $\left\{P_{n}(x)\right\}_{n=0}^{\infty}$, one of the most interesting problems in this subject is to study how the distribution of zeros of $P_{n}(x)$ is deformed when the coefficients of the recurrence relation satisfied by the orthogonal sequence are modified. It is a well-known fact that when standard modifications of the recurrence (associated, co-recursive, scaled co-recursive, co-modified, or any of its combinations) are considered, the asymptotic zero distribution of both the starting and perturbed families coincide. This means that, in this context, the finite $n$ case is the one of interest. In this situation, one of the most natural ways of dealing with this problem is to compute the moments of the zero distribution of $P_{n}(x)$ or, equivalently, the Newton sum rules of their zeros, i.e., the sum of the $k$-th power of the zeros, $k \geq 1$, from which valuable information can be derived (see, e.g., $[40,46])$.

Some of the well-known methods for determining Newton sum rules of the zeros of orthogonal polynomials [5-7, 12,36], semiclassical polynomials [45], and associated polynomials of any order [ 46 and the references therein] are based on the differential equations which are satisfied by these polynomials. In the case of co-recursive associated polynomials, to the best of our knowledge, only for the Laguerre polynomials [29] and for some special cases of Jacobi polynomials [30, 31] are the differential equations known. Another difficulty is that the known differential equations for the co-recursive associated polynomials are very complicated and difficult to handle. So methods based on the differential equations are not the natural way of dealing with these polynomials. Also some other well-known methods for determining Newton sum 
rules are based on the three-term recurrence relation, $[9,15,16]$, on the explicit expression of the polynomials [35], and on the weight function [10, 11 and the references therein]. For a summary of all these methods, see [44].

In Section 2, we determine the Newton sum rules $\sum_{n=0}^{N-1} \omega_{n}^{k}(\beta, \gamma, c), k=1,2, \ldots$, for the zeros $\omega_{n}(\beta, \gamma, c), n=0,1,2, \ldots, N-1$, of the scaled co-recursive associated polynomials $Q_{n}(x ; \beta, \gamma, c)$ in terms of the Newton sum rules $\sum_{n=0}^{N-1} \lambda_{n}^{k}(c), k=1,2, \ldots$, of the zeros $\lambda_{n}(c) \equiv \omega_{n}(0,1, c)$ of the corresponding associated polynomials $P_{n}(x ; c)$.

Some examples of Newton sum rules of the classical associated polynomials are given in Section 3.

In Section 4, we give some monotonicity properties of the zeros of the scaled corecursive associated polynomials.

\section{The main result}

The method we use is general and is valid also for other cases in addition to the particular case considered in this paper. It has been used successfully by the authors in previous work [20-26]. It is well-known (see for instance [25]) that $\omega_{n}(\beta, \gamma, c)$, $n=0,1,2, \ldots, N-1$ is a zero of the polynomial $Q_{n}(x ; \beta, \gamma, c)$, defined by $(1.4)$, if and only if it is an eigenvalue of the following generalized eigenvalue problem

$$
\left(T_{0}+\beta P_{0}\right) x_{n}=\omega_{n}(\beta, \gamma, c)\left(1+(\gamma-1) P_{0}\right) x_{n}, x_{n} \in H_{N}, \quad n=0,1,2, \ldots, N-1 .
$$

In (2.1), $T_{0}=A V^{*}+V A+B$ where $A, B$ are the diagonal operators $A e_{n}=\alpha_{n+c} e_{n}$, $B e_{n}=\beta_{n+c} e_{n}$ where $\alpha_{n+c}>0$ and $\beta_{n+c}$ are real sequences in a finite dimensional Hilbert space $H_{N}$ with the orthonormal basis $e_{n}, n=0,1,2, \ldots, N-1$ and scalar product $(\cdot, \cdot), V$ is the truncated shift operator $\left(V e_{n}=e_{n+1}, n=0,1, \ldots, N-2\right.$, $\left.V e_{N-1}=0\right)$, and $V^{*}$ is its adjoint $\left(V^{*} e_{n}=e_{n-1}, n=1,2, \ldots, N-1, V^{*} e_{0}=0\right)$. Finally $P_{0}$ is the projection operator on the one-dimensional space spanned by $e_{0}$, i.e., $P_{0} x=\left(x, e_{0}\right) e_{0}, x \in H_{N}$. The operator $C$ is defined by

$$
C e_{n}=c_{n} e_{n}
$$

where $c_{0}=\gamma$ and $c_{n}=1, n=1,2, \ldots, N-1$. Since $\gamma>0$, the operator $C$ is positive-definite, so that both $C^{-1}$ and $C^{-1 / 2}$ exist.

There is a simple and important result in operator theory which finds here a useful application. This result is known for operators of trace class and Hilbert-Schmidt operators. For a symmetric operator $M$ in a finite-dimensional real Hilbert space, e.g. the space $H_{N}$, the result can be stated as follows:

The sum $\sum_{n=0}^{N-1}\left(M e_{n}, e_{n}\right)$ is independent of the orthonormal basis $e_{n}$, $n=0,1,2, \ldots, N-1$, i.e., if $e_{n}$ and $v_{n}$ are two orthonormal bases in $H_{N}$, then

$$
\sum_{n=0}^{N-1}\left(M e_{n}, e_{n}\right)=\sum_{n=0}^{N-1}\left(M v_{n}, v_{n}\right) .
$$

If $x_{n}, n=0,1,2, \ldots, N-1$ is the complete orthonormal system of the eigenvectors of $T_{0}$ in $H_{N}$ and $\omega_{n}(0,1, c) \equiv \lambda_{n}(c)$ are the corresponding simple eigenvalues, i.e., the zeros of the polynomial $P_{n}(x ; c)$, then for $M=T_{0}^{k}$, we find from (2.1) that

$$
\sum_{n=0}^{N-1}\left(T_{0}^{k} e_{n}, e_{n}\right)=\sum_{n=0}^{N-1}\left(T_{0}^{k} x_{n}, x_{n}\right)=\sum_{n=0}^{N-1} \lambda_{n}^{k}(c) \text {. }
$$


From the relation,

$$
\begin{aligned}
& T_{0} e_{n}=\alpha_{n+c-1} e_{n-1}+\alpha_{n+c} e_{n+1}+\beta_{n+c} e_{n}, \\
& T_{0} e_{0}=\alpha_{0+c} e_{1}+\beta_{0+c} e_{0},
\end{aligned}
$$

we get for $1 \leq k \leq 4$

$$
\begin{aligned}
\sum_{n=0}^{N-1} \lambda_{n}(c)= & \sum_{n=0}^{N-1} \beta_{n+c}, \\
\sum_{n=0}^{N-1} \lambda_{n}^{2}(c)= & 2 \sum_{n=0}^{N-2} \alpha_{n+c}^{2}+\sum_{n=0}^{N-1} \beta_{n+c}^{2}, \\
\sum_{n=0}^{N-1} \lambda_{n}^{3}(c)= & 3 \sum_{n=0}^{N-2} \alpha_{n+c}^{2} \beta_{n+c}+3 \sum_{n=0}^{N-2} \alpha_{n+c}^{2} \beta_{n+c+1}+\sum_{n=0}^{N-1} \beta_{n+c}^{3}, \\
\sum_{n=0}^{N-1} \lambda_{n}^{4}(c)= & \sum_{n=0}^{N-1} \beta_{n+c}^{4}+2 \sum_{n=0}^{N-2} \alpha_{n+c}^{4}+4 \sum_{n=0}^{N-3} \alpha_{n+c}^{2} \alpha_{n+c+1}^{2}+4 \sum_{n=0}^{N-2} \alpha_{n+c}^{2} \beta_{n+c}^{2} \\
& +4 \sum_{n=0}^{N-2} \alpha_{n+c}^{2} \beta_{n+c+1}^{2}+4 \sum_{n=0}^{N-2} \alpha_{n+c}^{2} \beta_{n+c} \beta_{n+c+1} .
\end{aligned}
$$

The following theorem is useful in the case of scaled co-recursive associated polynomials where the differential equations in general are unknown or very difficult to handle.

Theorem 2.1. Let $\lambda_{n}(c)$ and $\omega_{n}(\beta, \gamma, c)$ be the zeros of the polynomials $P_{n}(x ; c)$ and $Q_{n}(x ; \beta, \gamma, c)$, respectively, and let $k$ be a positive integer. Then

$$
\sum_{n=0}^{N-1} \omega_{n}^{k}(\beta, \gamma, c)=\sum_{n=0}^{N-1} \lambda_{n}^{k}(c)+\left(T^{k} e_{0}, e_{0}\right)+\left(T^{k} e_{1}, e_{1}\right)-\left(T_{0}^{k} e_{0}, e_{0}\right)-\left(T_{0}^{k} e_{1}, e_{1}\right)
$$

where

$$
T=C^{-1 / 2}\left(T_{0}+\beta P_{0}\right) C^{-1 / 2}
$$

and

$$
T_{0}=A V^{*}+V A+B .
$$

Proof. By setting $x_{n}=C^{-1 / 2} y_{n}$ where $C=1+(\gamma-1) P_{0}$, the problem (2.1) is transformed to

$$
C^{-1 / 2}\left(T_{0}+\beta P_{0}\right) C^{-1 / 2} y_{n}=\omega_{n}(\beta, \gamma, c) y_{n}, \quad\left\|y_{n}\right\|=1 .
$$

From the above, we find

$$
T y_{n}=\omega_{n}(\beta, \gamma, c) y_{n}
$$

or

$$
\left(T y_{n}, y_{n}\right)=\omega_{n}(\beta, \gamma, c)
$$

and

$$
\sum_{n=0}^{N-1}\left(T y_{n}, y_{n}\right)=\sum_{n=0}^{N-1}\left(T e_{n}, e_{n}\right)
$$


So, from (2.12) and (2.13), we get

$$
\sum_{n=0}^{N-1} \omega_{n}(\beta, \gamma, c)=\sum_{n=0}^{N-1}\left(T e_{n}, e_{n}\right)
$$

and

$$
\sum_{n=0}^{N-1} \omega_{n}^{k}(\beta, \gamma, c)=\sum_{n=0}^{N-1}\left(T^{k} e_{n}, e_{n}\right), \quad k \geq 1
$$

or

$$
\sum_{n=0}^{N-1} \omega_{n}^{k}(\beta, \gamma, c)=\left(T^{k} e_{0}, e_{0}\right)+\left(T^{k} e_{1}, e_{1}\right)+\sum_{n=2}^{N-1}\left(T^{k} e_{n}, e_{n}\right)
$$

Since

$$
T=C^{-1 / 2} T_{0} C^{-1 / 2}+\beta C^{-1 / 2} P_{0} C^{-1 / 2},
$$

we obtain

$$
\begin{aligned}
& T e_{0}=C^{-1 / 2} T_{0} C^{-1 / 2} e_{0}+\frac{\beta}{\gamma} e_{0}, \\
& T e_{1}=C^{-1 / 2} T_{0} C^{-1 / 2} e_{1} \\
& T e_{n}=T_{0} e_{n}, \quad 2 \leq n \leq N-1,
\end{aligned}
$$

and from (2.14), we get (2.10) because

$$
\sum_{n=0}^{N-1} \lambda_{n}^{k}(c)=\sum_{n=0}^{N-1}\left(T_{0}^{k} e_{n}, e_{n}\right)
$$

Remark 2.1. For $1 \leq k \leq 4$, equation (2.10) gives for $k=1$ :

$$
\sum_{n=0}^{N-1} \omega_{n}(\beta, \gamma, c)=\sum_{n=0}^{N-1} \lambda_{n}(c)+\frac{\beta+\beta_{0+c}}{\gamma}-\beta_{0+c}
$$

for $k=2$ :

$$
\sum_{n=0}^{N-1} \omega_{n}^{2}(\beta, \gamma, c)=\sum_{n=0}^{N-1} \lambda_{n}^{2}(c)+\frac{\left(\beta+\beta_{0+c}\right)^{2}}{\gamma^{2}}+2 \alpha_{0+c}^{2}\left(\frac{1}{\gamma}-1\right)-\beta_{0+c}^{2},
$$

for $k=3$ :

$$
\begin{aligned}
\sum_{n=0}^{N-1} \omega_{n}^{3}(\beta, \gamma, c)= & \sum_{n=0}^{N-1} \lambda_{n}^{3}(c)+\frac{\left(\beta+\beta_{0+c}\right)^{3}}{\gamma^{3}}+\frac{3 \alpha_{0+c}^{2}}{\gamma^{2}}\left(\beta+\beta_{0+c}\right) \\
& +\frac{3 \alpha_{0+c}^{2} \beta_{1+c}}{\gamma}-\beta_{0+c}^{3}-3 \alpha_{0+c}^{2}\left(\beta_{0+c}+\beta_{1+c}\right),
\end{aligned}
$$


and for $k=4$ :

$$
\begin{aligned}
& \sum_{n=0}^{N-1} \omega_{n}^{4}(\beta, \gamma, c)=\sum_{n=0}^{N-1} \lambda_{n}^{4}(c)+\frac{\left(\beta+\beta_{0+c}\right)^{4}}{\gamma^{4}}+2 \alpha_{0+c}^{4}\left(\frac{1}{\gamma^{2}}-1\right) \\
& \quad+\frac{4 \alpha_{0+c}^{2}}{\gamma^{3}}\left(\beta_{0+c}+\beta\right)^{2}+\frac{2 \alpha_{0+c}^{2} \beta^{2}}{\gamma^{3}}+\frac{4 \alpha_{0+c}^{2} \beta_{1+c}^{2}}{\gamma}+\frac{4 \alpha_{0+c}^{2} \beta_{1+c}}{\gamma^{2}}\left(\beta+\beta_{0+c}\right) \\
& \quad+3\left(\frac{1}{\gamma}-1\right) \alpha_{0+c}^{2} \alpha_{1+c}^{2}-\beta_{0+c}^{4}-4 \alpha_{0+c}^{2}\left(\beta_{0+c}^{2}+\beta_{1+c}^{2}+\beta_{0+c} \beta_{1+c}\right)
\end{aligned}
$$

\section{Newton sum rules for the associated classical polynomials}

(1) Consider the associated Laguerre polynomials $L_{n}^{(a)}(x ; c), a>-1, c \geq 0$, which satisfy the recurrence relation

$$
\begin{gathered}
(n+c+1) L_{n+1}^{(a)}(x ; c)+(n+c+a) L_{n-1}^{(a)}(x ; c) \\
-(2 n+2 c+a+1) L_{n}^{(a)}(x ; c)=-x L_{n}^{(a)}(x ; c), \quad n=0,1,2, \ldots, \\
L_{-1}^{(a)}(x ; c)=0, \quad L_{0}^{(a)}(x ; c)=1 .
\end{gathered}
$$

Setting $L_{n}^{(a)}(x ; c)=(-1)^{n} \sqrt{U_{n}} Q_{n}^{(a)}(x ; c)$ where $U_{n}$ satisfies

$$
U_{-1}=0, \quad U_{0}=1, \quad U_{n}=\frac{n+c+a}{n+c} U_{n-1}, \quad n \geq 1,
$$

we obtain from (3.1)

$$
\begin{gathered}
\sqrt{(n+c+1)(n+c+a+1)} Q_{n+1}^{(a)}(x ; c)+\sqrt{(n+c+a)(n+c)} Q_{n-1}^{(a)}(x ; c) \\
+(2 n+2 c+a+1) Q_{n}^{(a)}(x ; c)=x Q_{n}^{(a)}(x ; c), \quad n \geq 1 \\
Q_{-1}^{(a)}(x ; c)=0, \quad Q_{0}^{(a)}(x ; c)=1 .
\end{gathered}
$$

The polynomials $Q_{n}^{(a)}(x ; c)$ and the associated Laguerre polynomials $L_{n}^{(a)}(x ; c)$ have the same zeros $\lambda_{n}(c)$. In this case, we have: $\alpha_{n+c}(a)=\sqrt{(n+c+1)(n+c+a+1)}$, $\beta_{n+c}(a)=(2 n+2 c+a+1), n=0,1,2, \ldots, N-1$, so, from the relations (2.6)-(2.9), we get:

$$
\begin{aligned}
\sum_{n=0}^{N-1} \lambda_{n}(c) & =N(N+2 c+a) \\
\sum_{n=0}^{N-1} \lambda_{n}^{2}(c) & =2 N^{3}+N^{2}(6 c+3 a-1)+N\left(6 c^{2}+6 c a-2 c+a^{2}-a\right)-2 c^{2}-2 c a \\
\sum_{n=0}^{N-1} \lambda_{n}^{3}(c) & =5 N^{4}+N^{3}(20 c+10 a-6)+N^{2}\left(30 c^{2}+30 a c-18 c+6 a^{2}-9 a+2\right) \\
+ & N\left(20 c^{3}+30 a c^{2}-18 c^{2}+12 a^{2} c-18 a c+4 c+a^{3}-3 a^{2}+2 a\right) \\
- & \left(12 c^{3}+18 a c^{2}+6 a^{2} c\right)
\end{aligned}
$$


and

$$
\begin{aligned}
\sum_{n=0}^{N-1} & \lambda_{n}^{4}(c)=14 N^{5}+N^{4}(70 c+35 a-29) \\
+ & N^{3}\left(140 c^{2}+140 a c-111 c+30 a^{2}-58 a+22\right) \\
+ & N^{2}\left(140 c^{3}+210 a c^{2}-174 c^{2}+90 a^{2} c-174 a c+66 c+10 a^{3}-35 a^{2}+33 a-6\right) \\
+ & N\left(70 c^{4}+140 a c^{3}-116 c^{3}+90 a^{2} c^{2}+174 a c^{2}-66 c^{2}+20 a^{3} c-70 a^{2} c-66 a c\right. \\
& \left.\quad-12 c+a^{4}-6 a^{3}+11 a^{2}-6 a\right) \\
& -\left(58 c^{4}+116 a c^{3}+70 a^{2} c^{2}+12 a^{3} c+12 a c\right) .
\end{aligned}
$$

(2) Consider the associated Hermite polynomials defined by

$$
\begin{gathered}
H_{n+1}(x ; c)+2(n+c) H_{n-1}(x ; c)=2 x H_{n}(x ; c), \quad n=0,1,2, \ldots, \\
H_{-1}(x ; c)=0, \quad H_{0}(x ; c)=1 .
\end{gathered}
$$

Setting $H_{n}(x ; c)=\sqrt{U_{n}} P_{n}(x ; c)$ where $U_{n}$ satisfies

$$
U_{-1}=0, \quad U_{0}=1, \quad U_{n}=2(n+c) U_{n-1}, \quad n \geq 1,
$$

we obtain from (3.7)

$$
\begin{gathered}
\sqrt{\frac{n+c+1}{2}} P_{n+1}(x ; c)+\sqrt{\frac{n+c}{2}} P_{n-1}(x ; c)=x P_{n}(x ; c), \\
P_{-1}(x ; c)=0, \quad P_{0}(x ; c)=1 .
\end{gathered}
$$

The $P_{n}(x ; c)$ and the associated Hermite polynomials $H_{n}(x ; c)$ have the same zeros $\lambda_{n}(c)$. In this case, we have $\alpha_{n+c}=\sqrt{\frac{n+c+1}{2}}, \beta_{n+c}=0, n=0,1,2, \ldots, N-1$, so, from the relations $(2.6)-(2.9)$, we get

$$
\sum_{n=0}^{N-1} \lambda_{n}(c)=\sum_{n=0}^{N-1} \lambda_{n}^{3}(c)=0
$$

because $\beta_{n+c}=0$,

$$
\sum_{n=0}^{N-1} \lambda_{n}^{2}(c)=\frac{(N-1)(N+2 c)}{2}
$$

and

$$
\sum_{n=0}^{N-1} \lambda_{n}^{4}(c)=\frac{1}{4}(N-1) N(2 N-3)+\frac{c}{2}(3 N-5)(N+c) .
$$

(3) Consider the associated Jacobi polynomials $P_{n}^{(a, b)}(x ; c)$, which are defined by

$$
\begin{gathered}
2(n+c+1)(n+c+a+b+1)(2 n+2 c+a+b) P_{n+1}^{(a, b)}(x ; c) \\
+2(n+c+a)(n+c+b)(2 n+2 c+a+b+2) P_{n-1}^{(a, b)}(x ; c) \\
+\left(b^{2}-a^{2}\right)(2 n+2 c+a+b+1) P_{n}^{(a, b)}(x ; c) \\
=(2 n+2 c+a+b+1)(2 n+2 c+a+b+2)(2 n+2 c+a+b) x P_{n}^{(a, b)}(x ; c), \\
P_{-1}^{(a, b)}(x ; c)=0, \quad P_{0}^{(a, b)}(x ; c)=1 .
\end{gathered}
$$


Setting $P_{n}^{(a, b)}(x ; c)=U_{n} R_{n}^{(a, b)}(x ; c)$ where

$$
\begin{gathered}
U_{-1}=0, \quad U_{0}=1, \\
U_{n+1}=\sqrt{\frac{(n+c+a+1)(n+c+b+1)(2 n+2 c+a+b+1)}{(n+c+1)(n+c+a+b+1)(2 n+2 c+a+b+3)}} U_{n},
\end{gathered}
$$

we find

$$
\begin{gathered}
\sqrt{\frac{4(n+c+1)(n+c+a+1)(n+c+b+1)(n+c+a+b+1)}{(2 n+2 c+a+b+2)^{2}(2 n+2 c+a+b+1)(2 n+2 c+a+b+3)}} R_{n+1}^{(a, b)}(x ; c) \\
+\sqrt{\frac{4(n+c)(n+c+a)(n+c+b)(n+c+a+b)}{(2 n+2 c+a+b)^{2}(2 n+2 c+a+b-1)(2 n+2 c+a+b+1)}} R_{n-1}^{(a, b)}(x ; c) \\
+\frac{\left(b^{2}-a^{2}\right)}{(2 n+2 c+a+b+2)(2 n+2 c+a+b)} R_{n}^{(a, b)}(x, c)=x R_{n}^{(a, b)}(x ; c), \\
R_{-1}^{(a, b)}(x ; c)=0, \quad R_{0}^{(a, b)}(x ; c)=1 .
\end{gathered}
$$

The polynomials $R_{n}^{(a, b)}(x ; c)$ and the associated Jacobi polynomials $P_{n}^{(a, b)}(x ; c)$ have the same zeros $\lambda_{n}(c)$. In this case, we have

$$
\begin{aligned}
& \alpha_{n+c}(a, b)=\sqrt{\frac{4(n+c+1)(n+c+a+1)(n+c+b+1)(n+c+a+b+1)}{(2 n+2 c+a+b+2)^{2}(2 n+2 c+a+b+1)(2 n+2 c+a+b+3)}}, \\
& \beta_{n+c}(a, b)=\frac{\left(b^{2}-a^{2}\right)}{(2 n+2 c+a+b+2)(2 n+2 c+a+b)}, \quad n=0,1,2, \ldots, N-1,
\end{aligned}
$$

and the relations (2.6), (2.7) give, respectively,

$$
\begin{gathered}
\sum_{n=0}^{N-1} \lambda_{n}(c)=\frac{N\left(b^{2}-a^{2}\right)}{(2 c+a+b)(2 N+2 c+a+b)} \\
\sum_{n=0}^{N-1} \lambda_{n}^{2}(c)=\frac{(N-1)[2 c(c+a+b+N)+N(a+b+1)]}{(2 c+a+b+1)(2 N+2 c+a+b-1)} \\
+\frac{(b-a)^{2}\left[(3 N-1)(b+a)^{2}(2 c+a+b)(2 N+2 c+a+b)\right]}{2(2 c+a+b+1)(2 N+2 c+a+b-1)(2 c+a+b)^{2}(2 N+2 c+a+b)^{2}} \\
-\frac{(b-a)^{2}\left[(N-1)(2 c+a+b)^{2}(2 N+2 c+a+b)^{2}+2 N^{2}(2 N-1)(b+a)^{2}\right]}{2(2 c+a+b+1)(2 N+2 c+a+b-1)(2 c+a+b)^{2}(2 N+2 c+a+b)^{2}} .
\end{gathered}
$$

Remark 3.1. For $c=0$, in (3.3)-(3.6), (3.10), (3.11), (3.14), and (3.15), we get the known [5,7] sum rules for the classical Laguerre, Hermite, and Jacobi polynomials, respectively, which were found using differential equations methods.

Remark 3.2. The sum rules (3.9), (3.10), (3.11) for the zeros of the associated Hermite polynomials have been found in [46], as an application of a program built in the Mathematica symbolic language. 


\section{Monotonicity properties of the zeros $\omega_{n}(\beta, \gamma, c)$}

The operator (2.11) can be written

$$
T=C^{-1 / 2} T_{0} C^{-1 / 2}+\beta C^{-1 / 2} P_{0} C^{-1 / 2} .
$$

The eigenvalues $\omega_{n}(\beta, \gamma, c)$ of $T$, i.e., the zeros of the polynomial $Q_{n}(x ; \beta, \gamma, c)$, are differentiable functions of $\beta$ and the derivatives are given [20] by

$$
\frac{\partial \omega_{n}(\beta, \gamma, c)}{\partial \beta}=\left(C^{-1 / 2} P_{0} C^{-1 / 2} y_{n}, y_{n}\right)=\left|\left(C^{-1 / 2} y_{n}, e_{0}\right)\right|^{2}=\left|\left(y_{n}, C^{-1 / 2} e_{0}\right)\right|^{2}
$$

where $y_{n}, n=0,1,2, \ldots, N-1$, are the normalized eigenvectors of $T$ which correspond to the eigenvalues $\omega_{n}(\beta, \gamma, c): T y_{n}=\omega_{n}(\beta, \gamma, c) y_{n}$.

The inequality

$$
\frac{\partial \omega_{n}(\beta, \gamma, c)}{\partial \beta} \leq \frac{1}{\gamma}
$$

follows immediately from (4.1) because $\left\|y_{n}\right\|=1$ and $\left\|C^{-1 / 2} e_{0}\right\|^{2}=\frac{1}{\gamma}$. We also observe that

$$
\begin{aligned}
\frac{\partial}{\partial \beta}\left(\frac{\omega_{n}(\beta, \gamma, c)}{\beta}\right) & =\frac{\beta \frac{\partial \omega_{n}(\beta, \gamma, c)}{\partial \beta}-\omega_{n}(\beta, \gamma, c)}{\beta^{2}}=-\frac{1}{\beta^{2}}\left(C^{-1 / 2} T_{0} C^{-1 / 2} y_{n}, y_{n}\right) \\
& =-\frac{1}{\beta^{2}}\left(T_{0} x_{n}, x_{n}\right)
\end{aligned}
$$

where $x_{n}=C^{-1 / 2} y_{n}$.

If $\lambda_{0}(c)$ is the smallest zero of $P_{n}(x ; c)$, i.e., the first eigenvalue of $T_{0}$, then the operator $T_{0}+\lambda_{0}(c)$ is positive, i.e.,

$$
\left(\left(T_{0}+\lambda_{0}(c)\right) f, f\right) \geq 0
$$

for every $f \in H_{N}$. Thus from (4.3), we find

$$
\frac{\partial}{\partial \beta}\left(\frac{\omega_{n}(\beta, \gamma, c)}{\beta}\right) \leq \frac{\lambda_{0}(c)}{\beta^{2}}\left\|x_{n}\right\|^{2} \leq \frac{\lambda_{0}(c)}{\beta^{2}}\left\|C^{-1 / 2}\right\|^{2} .
$$

From the above, we obtain the following results:

(i) The function $\omega_{n}(\beta, \gamma, c)$ increases with $\beta$. This follows from (4.1).

(ii) The function $\omega_{n}(\beta, \gamma, c)-\frac{\beta}{\gamma}$ decreases with $\beta>0$. This follows from (4.2) because

$$
\frac{\partial}{\partial \beta}\left(\omega_{n}(\beta, \gamma, c)-\frac{\beta}{\gamma}\right) \leq 0
$$

(iii) If $\lambda_{0}(c) \leq 0$, then the function $\frac{\omega_{n}(\beta, \gamma, c)}{\beta}$ decreases with $\beta>0$. This follows immediately from (4.4).

(iv) If $\lambda_{0}(c)>0$, then the functions $\frac{\gamma \omega_{n}(\beta, \gamma, c)+\lambda_{0}(c)}{\beta}$ and $\frac{\omega_{n}(\beta, \gamma, c)+\lambda_{0}(c)}{\beta}$ decrease with $\beta>0$ for $0<\gamma \leq 1$ and for $\gamma>1$, respectively. This follows from (4.4) because $\left\|C^{-1 / 2}\right\|^{2}=\frac{1}{\gamma}$ for $0<\gamma \leq 1$ and $\left\|C^{-1 / 2}\right\|^{2}=1$ for $\gamma>1$.

Remark 4.1. From (4.5) we have for $\beta>0$

$$
\omega_{n}(\beta, \gamma, c)<\omega_{n}(0, \gamma, c)+\frac{\beta}{\gamma}
$$


This inequality gives an upper bound for all the zeros $\omega_{n}(\beta, \gamma, c), n=0,1,2, \ldots, N-$ 1 of the scaled co-recursive associated polynomials, and the bound is sharp for small $\beta$ because for $\beta=0,(4.6)$ becomes an equality.

Remark 4.2. From (4.1) we obtain

$$
\frac{\partial \omega_{n}(\beta, \gamma, c)}{\partial \beta}=\frac{1}{\gamma}\left|\left(y_{n}, e_{0}\right)\right|^{2}
$$

and

$$
\sum_{n=0}^{N-1} \frac{\partial \omega_{n}(\beta, \gamma, c)}{\partial \beta}=\frac{1}{\gamma} \sum_{n=0}^{N-1}\left|\left(y_{n}, e_{0}\right)\right|^{2}=\frac{1}{\gamma}\left\|e_{0}\right\|^{2}=\frac{1}{\gamma} .
$$

This means that

$$
\frac{\partial}{\partial \beta}\left(\sum_{n=0}^{N-1} \omega_{n}(\beta, \gamma, c)-\frac{\beta}{\gamma}\right)=0 .
$$

Thus we obtain the relation

$$
\sum_{n=0}^{N-1} \omega_{n}(\beta, \gamma, c)-\frac{\beta}{\gamma}=\sum_{n=0}^{N-1} \omega_{n}(0, \gamma, c),
$$

which follows also from the relation (2.16).

Acknowledgements. We thank the referees for their helpful remarks and suggestions and also for bringing references $[9,10,35]$ to our attention.

\section{References}

1. W. R. Allaway, The identification of a class of orthogonal polynomial sets, $\mathrm{PhD}$ Thesis, University of Alberta, Canada, 1972.

2. R. Askey and J. Wimp, Associated Laguerre and Hermite polynomials, Proc. Roy. Soc. Edinburgh 96 (1984), 15-37.

3. S. Belmehdi and A. Ronveaux, Polynomes associes des polynomes orthogonaux classiques. Construction via Reduce, Actas del VI Simposium on Polinomios Orthogonales y Aplicationes (1989), pp.72-83, Gijon, Spain.

4. , The fourth-order differential equation satisfied by the associated orthogonal polynomials, Rend. Math. Appl. 11 (1991), 313-326.

5. E. Buendia, J. S. Dehesa, and M. A. Sanchez-Buendia, On the zeros of eigenfunctions of polynomial differential operators, J. Math. Phys. 26 (1985), 2729-2736.

6. E. Buendia, J. S. Dehesa, and F. J. Galvez, The distribution of zeros of the polynomial eigenfunctions of ordinary differential operators of arbitrary order, Lect. Notes in Math. 1329 (1988), 222-235.

7. K. M. Case, Sum rules for the zeros of polynomials I, J.Math. Phys. 21 (1980), 702-708.

8. T. S. Chihara, On co-recursive orthogonal polynomials, Proc. Amer. Math. Soc. 8 (1957), 899905.

9. J. S. Dehesa, On the conditions for a Hamiltonian matrix to have an eigenvalue density with some prescribed characteristics, J. Comput. Appl. Math. 2 (1976), 249-254.

10. The spectrum of Jacobi matrices in terms of its associated weight function, J. Comput. Appl. Math. 4 (1978), 275-283.

11. (1979), 277-284.

12. J. S. Dehesa, E. Buendia, and M. A. Sanchez-Buendia, On the polynomial solutions of ordinary differential equations of the fourth order, J. Math. Phys. 26 (1985), 1547-1552.

13. E. A. van Doorn, On a class of generalized orthogonal polynomials with real zeros, in IMACS, Orthogonal Polynomials and Their Applications, pp.231-236, 1991.

14. 309-317. 
15. B. Germano and P. E. Ricci, Representation formulas for the moments of the density of zeros of orthogonal polynomials, Mathematiche (Catania) 48 (1993), 77-86.

16. B. Germano, P. Natalini, and P. E. Ricci, Computing the moments of the density of zeros for orthogonal polynomials, Computers Math. Appl., Special issue on Concrete Analysis, 30 (1995), 69-81.

17. C. C. Grosjean, Theory of recursive generation of systems of orthogonal polynomials: An illustrative example, J. Comput. Appl. Math. 12, 13 (1985), 299-318.

18. - The weight functions, generating functions and miscellaneous properties of the sequences of orthogonal polynomials of the second kind associated with Jacobi and the Gegenbauer polynomials, J. Comput. Appl. Math. 16 (1986), 299-318.

19. W. Hahn, Uber Orthogonalpolynome mit drei parameteren, Deutsche Math 5 (1940-41), 373-378.

20. E. K. Ifantis, $A$ theorem concerning differentiability of eigenvectors and eigenvalues with some applications, Appl. Anal. 28 (1988), 257-283.

21. E. K. Ifantis and P. N. Panagopoulos, On the zeros of a class of polynomials defined by a three term recurrence relation, J. Math. Anal. Appl. 182 (1994), 361-370.

22. E. K. Ifantis and P. D. Siafarikas, Differential inequalities on the greatest zero of Laguerre and ultraspherical polynomials, Actas del VI Simposium on Polinomios Orthogonales y Aplicationes, pp.187-197, Gijon, Spain, 1989.

23. , On the zeros of a class of polynomials including the generalized Bessel polynomials, in: Orthogonal Polynomials and Their Applications (Ed. J. S. Dehesa), J. Comput. Appl. Math. 49 (1993), 103-109.

24. and Hermite polynomials, International Joint Symposium on Special Functions and Artificial Intelligence, Torino, Italy, 1993; Annals of Numerical Mathem. 2 (1995), 79-91.

25. _ Perturbation of the coefficients in the recurrence relation of a class of polynomials, 4 th International Symposium on Orthogonal Polynomials and Their Applications, Evian, France, 1992; J. Comput. Appl. Math. 57 (1995), 163-170.

26. __ An alternative proof of a theorem of Stieltjes and related results, J. Comput. Appl. Math. 65 (1995), 165-172.

27. M. E. H. Ismail, D. R. Masson, J. Letessier, and G. Valent, Birth and death processes and orthogonal polynomials, in Orthogonal Polynomials: Theory and Practice (Ed. P. Nevai), pp.229255, Kluwer, Dordrecht, 1990.

28. M. E. H. Ismail and M. E. Muldoon, A discrete approach to monotonicity of zeros of orthogonal polynomials, Trans. Amer. Math. Soc. 323, (1991), 65-78.

29. J. Letessier, Co-recursive associated Laguerre polynomials, VII Simposium sobre Polinomios Orthogonales Aplicaciones, Granada, 1991; J. Comput. Appl. Math. 49 (1993), 127-136.

30. Some results on co-recursive associated Laguerre and Jacobi polynomials, SIAM J. Math. Anal. 25 (1994), 528-548.

31. On co-recursive associated Jacobi polynomials, 4th International Symposium on Orthogonal Polynomials and Their Applications, Evian, France, 1992; J. Comput. Appl. Math. 57, (1995), 203-213.

32. S. Lewanowicz, Results on the associated Jacobi and Gegenbauer polynomials, in: Orthogonal Polynomials and Their Applications (Ed. J. S. Dehesa), J. Comput. Appl. Math., 49 (1993), 137-143.

33. F. Marcellan, J. S. Dehesa, and A. Ronveaux, On orthogonal polynomials with perturbed recurrence relations, J. Comput. Appl. Math. 30 (1990), 203-212.

34. F. Peherstorfer, Finite perturbations of orthogonal polynomials, J. Comput. Appl. Math. 44 (1992), 275-302.

35. I. V. V. Raghanacharyulu and A. R. Tekumalla, Solution of the difference equations of generalized Lucas polynomials, J. Math. Phys. 13 (1972), 321-324.

36. P. E. Ricci, Sum rules for the zeros of polynomials and generalized Lucas polynomials, J. Math. Phys. 34 (1993), 4884-4891.

37. A. Ronveaux, Fourth-order differential equations for numerator polynomials, J. Phys. A 21 (1988), 749-753.

38. _ Fourth-order differential equations and orthogonal polynomials of the Laguerre-Hahn class, in: Orthogonal Polynomials and Their Applications (Eds. C. Brezinski, L. Gori, and A. Ronveaux), pp.379-385, IMACS Ann. Comput. Appl. Math. 9, Baltzer, Basel, 1991.

39. A. Ronveaux and F. Marcellan, Co-recursive orthogonal polynomials and fourth-order differential equation, J. Comput. Appl. Math. 25 (1989), 105-109. 
40. A. Ronveaux, A. Zarzo, and E. Godoy, Fourth-order differential equations satisfied by the generalized co-recursive of all classical orthogonal polynomials. A study of their distribution of zeros, J. Comput. Appl. Math. 59 (1995), 295-328.

41. K. I. Sato, On zeros of a system of polynomials and application to sojourn time distributions of birth-and-death processes, Trans. Amer. Math. Soc. 309 (1988), 375-390.

42. H. A. Slim, On co-recursive orthogonal polynomials and their application to potential scattering, J. Math. Anal. Appl. 136 (1988), 1-19.

43. J. Wimp, Explicit formulas for the associated Jacobi polynomials and some applications, Canad. J. Math. 39 (1987), 983-1000.

44. A. Zarzo, Estudio de las densidades discreta y asintotica de ceros de polinomios ortogonales, Tesina de Licenciatura, Univ. Granada, 1990.

45. A. Zarzo, J. H. Dehesa, and A. Ronveaux, Newton sum rules of zeros of semiclassical orthogonal polynomials, J. Comput. Appl. Math. 33, (1990), 85-96.

46. A. Zarzo, A. Ronveaux, and E. Godoy, Fourth-order differential equation satisfied by the associated of any order of all classical orthogonal polynomials. A study of their distribution of zeros, in: Orthogonal Polynomials and their Applications (Ed. J. S. Dehesa), J. Comput. Appl. Math. 49 (1993), 349-359.

Department of Mathematics, University of Patras, Patras, Greece 